\title{
MicroRNA-100 promotes migration and invasion through mammalian target of rapamycin in esophageal squamous cell carcinoma
}

\author{
NAIJIAN ZHANG ${ }^{1,2^{*}}$, HAILONG FU ${ }^{1,4^{*}}$, LEILEI SONG ${ }^{1}$, YOUHONG DING ${ }^{3}$, \\ XIUFANG WANG $^{1,2}$, CHAO ZHAO $^{1}$, YIQI ZHAO ${ }^{3}$, \\ FENG JIAO $^{3}$ and YAPING ZHAO ${ }^{1}$
}

\begin{abstract}
${ }^{1}$ Department of Laboratory Medicine, The 82nd Hospital of the People's Liberation Army, Huaian 223001;
${ }^{2}$ Department of Postgraduates, Bengbu Medical College, Bengbu 233030; ${ }^{3}$ Department of General Surgery, The 82nd Hospital of the People's Liberation Army, Huaian 223001; ${ }^{4}$ Key Laboratory of Laboratory Medicine of Jiangsu Province, Medical School, Jiangsu University, Zhenjiang, Jiangsu 212013, P.R. China
\end{abstract}

Received April 3, 2014; Accepted May 23, 2014

DOI: $10.3892 /$ or.2014.3389

\begin{abstract}
Esophageal squamous cell carcinoma (ESCC) is the predominant histologic subtype of esophageal cancer and is characterized by a high mortality rate and geographic differences in incidence. microRNAs (miRNAs) are small, non-coding RNAs that play important roles in the regulation of genes associated with cancer development and progression. In the present study, we demonstrated that microRNA-100 (miR-100) demonstrated markedly lower expression in the ESCC tissues as validated by quantitative reverse transcriptionpolymerase chain reaction (qRT-PCR). Furthermore, we found that the downregulation of miR-100 was significantly correlated with the status of lymph node metastasis in the 34 ESCC patients. Next, we investigated the role and mechanism of miR-100 in ESCC cells and found that miR-100 modulated the migration and invasion but not the apoptosis and proliferation of ESCC cells in vitro. We further demonstrated that miR-100 directly targeted the mTOR 3'UTR and repressed the expression of mTOR, a tumor-related gene. Similarly, miR-100 has been reported as a tumor suppressor by controlling cell migration and invasion, as it can target mTOR genes. These results provide insight into the potential mechanisms of miR-100 in the pathogenesis of ESCC.
\end{abstract}

Correspondence to: Dr Yaping Zhao, Department of Laboratory Medicine, The 82nd Hospital of the People's Liberation Army, Huaian 223001, P.R. China

E-mail: zyp82yy@aliyun.com

Dr Feng Jiao, Department of General Surgery, The 82nd Hospital of the People's Liberation Army, Huaian 223001, P.R. China

E-mail: jiaofeng7701@163.com

${ }^{*}$ Contributed equally

Key words: esophageal squamous cell carcinoma, microRNA, miR-100, invasion, metastasis, bioinformatic analysis

\section{Introduction}

Esophageal cancer (EC) occurs worldwide with a variable geographic distribution and ranks eighth in order of occurrence and is the sixth leading cause of cancer-related mortality (1). According to the etiologic and pathologic characteristics, it is divided into two main subtypes: esophageal squamous cell carcinoma (ESCC) and esophageal adenocarcinoma (EAC). In contrast to that of Western countries, ESCC is the most prevalent type of EC in China. ESCC is often diagnosed at later stages, so that the prognosis of affected patients is unsatisfactory, despite the development of therapeutic options such as surgery, chemotherapy and radiotherapy (2). The overall survival for advanced and metastatic cancer remains dismal, with a 5-year survival rate of less than $30 \%$ (3). Nevertheless, to date, efforts to indentify molecular markers associated with the pathogenesis of ESCC have elucidated only a few molecular mechanisms.

MicroRNAs (miRNAs) are a class of non-coding small RNAs that regulate gene expression at the post-transcriptional level by mainly binding to 3'UTR of target mRNAs, leading to mRNA degradation or translation inhibition (4). It has been predicted that approximately one-third of human genes are regulated by miRNAs (5). An aberrant miRNA expression signature is a hallmark of several diseases, including cancer (6-9). More than 50\% of annotated human miRNA genes are located in fragile chromosomal regions that are susceptible to amplification, deletion or translocation during the process of tumor development and can function either as oncogenes or tumor suppressors (10). A growing body of evidence has recently identified a considerable number of dysregulated miRNAs which may play a pivotal role in the pathogenesis of ESCC. Kong et al (11) demonstrated that miR-375 has a strong tumor-suppressive effect by inhibiting the expression of IGF1R. Downregulation of miR-375, which is mainly caused by promoter methylation, is one of the molecular mechanisms involved in the development and progression of ESCC. The results of Zhang et al demonstrated that miR-200b modu- 
lates the actin cytoskeleton (12), focal adhesion formation and the migratory/invasive properties of ESCC via targeting Kindlin-2. In our previous study, we identified 29 miRNAs with upregulated expression and 33 miRNAs with downregulated expression in ESCC tissues compared with the corresponding non-tumor tissues (13). microRNA-100 (miR-100) was one of the 33 downregulated miRNAs. miR-100 has been reported to be involved in tumorigenesis and tumor progression of several cancer types, including prostate and lung cancer, acute myeloid leukemia, epithelial ovarian, bladder, oral and breast cancer (14-20). However, the mechanism underlying the involvement of miR-100 in ESCC has not yet been clarified. In the present study, we found that miR-100 expression was significantly correlated with the status of lymph node metastasis in ESCC. miR-100 inhibited ESCC cell migration and invasion by downregulating the mTOR pathway.

\section{Materials and methods}

Clinical ESCC specimens and ESCC cell culture. Thirty-four pairs of primary ESCC and corresponding normal esophageal epithelial tissue sections were obtained from patients who had undergone surgery at the Cancer Center of The 82nd Hospital of the People's Liberation Army from 2008 to 2010. None of the patients received chemotherapy or radiotherapy before surgery. The excised specimens were quickly frozen and stored in liquid nitrogen until the extraction of RNA. Histological confirmation of the ESCC diagnosis was performed by a pathologist. The clinicopathological information for all patients is documented in our previous study (13). A total of 34 ESCC samples, subdivided according to clinicopathologic classification by age ( $\leq 60$ years, $n=18 ;>60$ years, $n=16$ ), by gender (males, $n=27$; females, $n=7$ ), by grade of differentiation (well, $n=12$; moderate, $n=17$; poor, $n=5$ ), by the degree of tumor invasion (submucosa, $n=3$; muscularis propria, $n=30$; adventitia, $n=1$ ), by lymph node metastasis (negative, $n=26$; positive, $n=8$ ) (13) were used in the present study. This study was approved by the Ethics Committee of The 82nd Hospital, and informed consent was obtained from each patient.

The human ESCC cell lines ECA109 (well differentiated) and TE-1 (moderately differentiated) were obtained from the Shanghai Institute of Cell Biology, Chinese Academy of Sciences and the KYSE150 cell line (poorly differentiated) was obtained from the American Type Culture Collection (ATCC). Human gastrointestinal tract cancer cell lines HEPG2 (liver hepatocellular carcinoma cells), HT-29 (colon adenocarcinoma cells) and AGS (gastric adenocarcinoma cells) were provided by the Institute of Pediatrics, Nanjing Medical University, Nanjing, China. ESCC cell lines were cultured in RPMI-1640 medium and gastrointestinal tract cancer cell lines were cultured in DMEM, and (for each cell line) supplemented with $10 \%$ (v/v) fetal bovine serum, $100 \mathrm{U} / \mathrm{ml}$ penicillin and $100 \mathrm{mg} /$ $\mathrm{ml}$ streptomycin at $37^{\circ} \mathrm{C}$ in a humidified incubator containing $5 \% \mathrm{CO}_{2}$.

miRNA isolation. Total RNA was isolated from tissue specimens using the mirVana miRNA isolation kit (Ambion, Austin, TX, USA), which allows for capture of both enriched miRNA and larger RNA species separately. Both the enriched miRNA and large RNA were quantified using a Nanodrop spectro- photometer and the integrity of the large RNA fraction was determined using an Agilent Bioanalyzer as a surrogate for miRNA quality control. Samples of miRNA were only used in the microarray experiments if the RNA integrity number (RIN) was 6 for the large RNA from the same specimen.

TaqMan miRNA assays. Mature miRNA quantification was performed using TaqMan miRNA analysis for miR-100. A mixture of $70 \mathrm{ng}$ of total RNA, $3.33 \mathrm{U} / \mathrm{ml}$ MultiScribe reverse transcriptase (RT), $50 \mathrm{nM}$ miRNA-specific stem-loop RT primer, RT buffer, $0.25 \mathrm{mM}$ each dNTP and $0.25 \mathrm{U} / \mathrm{ml}$ RNase inhibitor was used in a $15 \mu \mathrm{l}$ volume for $30 \mathrm{~min}$ at $16^{\circ} \mathrm{C}, 30 \mathrm{~min}$ at $42^{\circ} \mathrm{C}$ and $5 \mathrm{~min}$ at $85^{\circ} \mathrm{C}$ using the TaqMan microRNA reverse transcription kit (Applied Biosystems, Foster City, CA, USA). For real-time PCR, $1 \mu \mathrm{l}$ of cDNA, $10 \mu 1$ of TaqMan 2X Universal PCR Master Mix without uracil-N-glycosylase, $1 \mu \mathrm{l}$ of the TaqMan miRNA assay mix (Applied Biosystems) and $8 \mu \mathrm{l}$ of water were mixed for $10 \mathrm{~min}$ at $95^{\circ} \mathrm{C}$ followed by 40 cycles of $15 \mathrm{sec}$ at $95^{\circ} \mathrm{C}$ and $1 \mathrm{~min}$ at $60^{\circ} \mathrm{C}$. All real-time PCR experiments were performed using an ABI 7500 real-time PCR system (Applied Biosystems). The real-time PCR results were analyzed and expressed as relative miRNA expression of the threshold cycle $(\mathrm{Ct})$ value. The RT primer, PCR primers and TaqMan probe for miRNA were purchased from Applied Biosystems. U6 RNA was used for normalization.

miR-100 plasmid construction and the miR-100 inhibitor. The miR-100 expression vector was constructed by cloning of annealed oligonucleotides that contained the pre-miR100 sequence into the CSII-EF-cherry-MCS expression vector which were provided by the Institute of Pediatrics, Nanjing Medical University, Nanjing, China. A scrambled sequence was used as a negative control. miR-100 inhibitor (GenePharma Inc., Shanghai, China) was used to knock down miR-100 expression and the non-specific miRNA inhibitor was used as an appropriate negative control. The expression vector or inhibitor were transfected into the KYSE150 or ECA109 cell line, respectively, using Lipofectamine 2000 (Invitrogen, Carlsbad, CA, USA) according to the manufacturer's instructions.

Wound healing and Transwell migration assays. KYSE150 and ECA109 cells were transfected with the miR-100-vector or -inhibitor, respectively. After the cells reached subconfluence, wounds were created using a $200-\mu l$ pipette tip. The cells were then rinsed with medium to remove any free-floating cells and debris. Medium was then added, and the culture plates were incubated at $37^{\circ} \mathrm{C}$. Wound healing was observed at different time points within the scrape line, and representative scrape lines were photographed (magnification, x100). Duplicate wells for each condition were examined, and each experiment was repeated three times. For the migration assay, $5 \times 10^{4}$ ECA109 and KYSE150 cells were plated onto 24-well Transwell plates (Millipore, Billerica, MA, USA) with an $8-\mu \mathrm{m}$ pore polycarbonate membrane. For the invasion assay, $5 \times 10^{5}$ ECA109 and KYSE150 cells were plated on chambers precoated with $20 \mathrm{~g}$ of Matrigel. In both assays, the cells were plated in RPMI-1640 medium without serum and medium containing $10 \%$ fetal bovine serum in the lower chamber. After 
24 or $36 \mathrm{~h}$, the cells that did not migrate or invade through the pores were removed by cotton swabs. The cells on the filter surface were fixed with $4 \%$ formaldehyde, stained with $0.5 \%$ crystal violet and examined under a microscope. The invaded cells were counted under microscopic observation at a magnification of $x 400$. Three random microscopic fields were examined in each well and the counts were averaged.

Cell proliferation and apoptosis assays. ECA109 and KYSE-150 cell suspensions were plated into 96-well plates and were pre-incubated in a humidified incubator at $37^{\circ} \mathrm{C}$ in $5 \% \mathrm{CO}_{2}$ for $24 \mathrm{~h}$ before the transfection. At $0,12,24,48$ and $72 \mathrm{~h}$ after the transient transfection, $10 \mu \mathrm{l}$ of Cell Counting Kit- 8 solution (CCK-8, Dojindo Laboratories, Kumamoto, Japan) was added to each well in the plate, and then the plate was incubated for $1 \mathrm{~h}$ before measuring the absorbance at $450 \mathrm{~nm}$ in a microplate reader. Apoptosis was tested using the PE Annexin V apoptosis detection kit (BD Pharmingen, San Diego, CA, USA). Forty-eight hours after transfection, $4 \times 10^{5}$ cells were incubated with PE Annexin V and 7-AAD for $15 \mathrm{~min}$ at room temperature and then analyzed by Becton-Dickinson FACScan flow cytometer (FACScan, Becton-Dickinson, San Jose, CA, USA).

Bioinformatic analysis. Bioinformatics analysis available on the Web, including miRBase, NCBI mapviewer, UCSC Genome Browser was used. The miR-100 targets predicted by computer-aided algorithms were obtained from Pictar (http://pictar.bio.nyu.edu/), Targetscan (http://www.targetscan. org/) and miRanda (http://www.microrna.org/).

Luciferase reporter assay. The psiCHECK-2 system (Applied Biosystems) was utilized to demonstrate the direct targets of miR-100 in vitro. A truncated fragment (448 bp) of the 3'UTR of mTOR mRNA that contains the predicted microRNA binding site was cloned (forward primer, 5'-CCGCTCGAG TGGTGCCCTTTCTGGTAA-3' and reverse primer, 5'-ATT TGCGGCCGCAGGCTTCTTGGCTGTGCT-3') out of the genome and into the $X h o \mathrm{I}$ and Not I restriction sites in the psiCHECK-2 vector to establish the psiCHECK-2-mTOR-WT vector. As a control, a 448-bp double-stranded fragment containing a mutated version of the predicted binding site was synthesized. A Renilla luciferase expression construct served as the internal control (Promega, Madison, WI, USA) in this study. The luciferase activity was measured using a dual-luciferase reporter system (Promega) and an LB 960 Centro microplate luminometer (Berthold, Bad Wildbad, Germany). The intensity of the luminescence from the firefly luciferase was normalized to that of the Renilla luciferase.

Immunohistochemistry analysis. Immunohistochemistry was carried out to examine mTOR and IGF1R expression in 34 human ESCC and normal tissue specimens. mTOR and IGF1R were detected using a rabbit monoclonal antibody and antibodies against IGF1R (Cell Signaling Technology). Briefly, paraffin sections of the ESCC tissues from the patients were deparaffinized with xylene and rehydrated. Antigenic retrieval was processed by submerging the samples in citrate buffer ( $\mathrm{pH} \mathrm{6.0)}$ ) and microwaving. The sections were then treated with $3 \%$ hydrogen peroxide in methanol to quench the endogenous peroxidase activity followed by incubation with
$1 \%$ bovine serum albumin to block the non-specific binding. The sections were then stained with the anti-mTOR antibody (1:50) and anti-IGF1R antibody for $90 \mathrm{~min}$ at room temperature. After washing, the tissue sections were then incubated with the biotinylated anti-mouse secondary antibody followed by further incubation with streptavidin-horseradish peroxidase complex. The tissue sections were immersed in 3-amino9-ethyl carbazole and counterstained with 10\% Mayer's hematoxylin, dehydrated and mounted in crystal mount. For the negative control, the primary antibody was replaced by the non-immune mouse IgG of the same isotype. The degree of immunostaining of the formalin-fixed, paraffin-embedded sections was evaluated by two independent observers and moderate to strong nuclear staining was scored as a positive reaction. The distribution of $\mathrm{mTOR}$ and IGF1R were scored as follows: negative ( $<10 \%$ of the cells were positive) and positive (where $\geq 10 \%$ of the cells were positive).

Western blot analysis. ECA109 cells were transfected with miR-100-vector/inhibitor and protein lysates were separated by $10 \%$ SDS-PAGE, and electrophoretically transferred to a PVDF (polyvinylidene difluoride) membrane (Millipore). Then, the membrane was incubated with the rabbit monoclonal antibody against human mTOR (Cell Signaling Technology, \#2983), FZD8 (Abcam, ab75235), IGF1R (Abcam, ab39675), FGFR3 (Cell Signaling Technology, \#4574) followed by horseradish peroxidase (HRP)-labeled goat anti-rabbit IgG (Santa Cruz Biotechnology) and detected by chemiluminescence. $\beta$-actin (Bioworld Technology, Inc.) was used as a protein loading control. The intensity of protein fragments was quantified using Bio-Rad software.

Statistical analysis. The analysis of variance was used to determine the significance of differences between paired data sets. Values are presented as mean \pm standard deviation (SD). A $\mathrm{P}<0.05$ was considered to indicate a statistically significant result, which is indicated by an asterisk in the figures.

\section{Results}

miR-100 is significantly downregulated in ESCC. In our previous microarray study (13), we reported that miR-100 was significantly downregulated in ESCC tissues with a $>8$-fold decrease in ESCC compared to the corresponding non-tumor tissues. To confirm the microarray analysis results, we determined miR-100 expression using real-time quantitative reverse transcription PCR (qRT-PCR). which showed results similar to those in the microarray analysis (Fig. 1A).

We performed qRT-PCR on the reverse transcription product of the miRNAs that were extracted from 34 pairs of ESCC tissue samples. The relative expression levels of miR-100 in the ESCC tissues and the corresponding nontumor tissues are shown in Fig. 1B. Of the 34 ESCC tissues, there were 28 cases with downregulation of miR-100 when compared with the corresponding non-tumor tissues, with a difference in expression <2-fold (11 cases, $0.32 \%$ ), $>2$-fold (4 cases, $0.12 \%$ ), >3-fold (13 cases, $0.38 \%$ ). In contrast, miR-100 was upregulated in 6 cases of ESCC $(0.18 \%)$ when compared to the corresponding non-tumor tissues (all with a difference in expression $<1$-fold). We next detected the expres- 
A

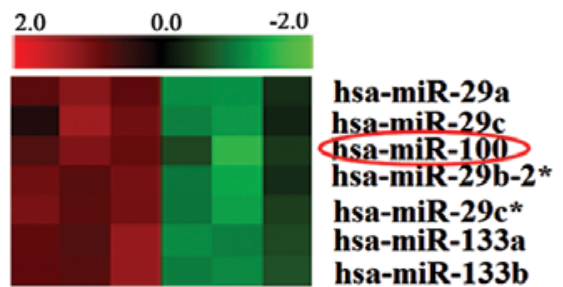

B

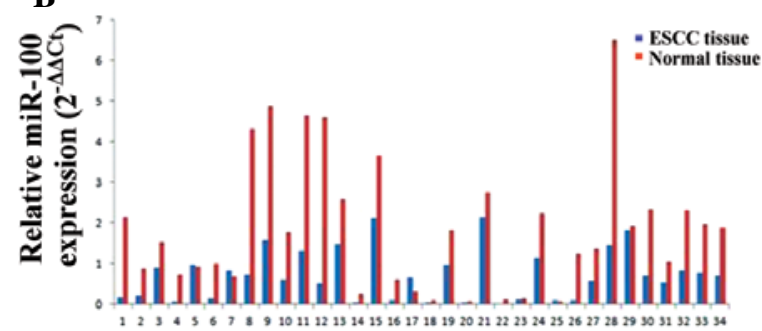

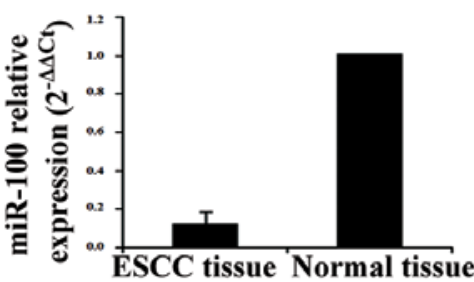

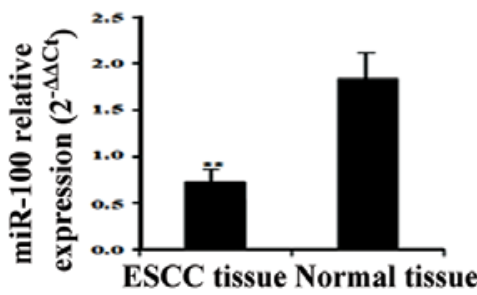

C

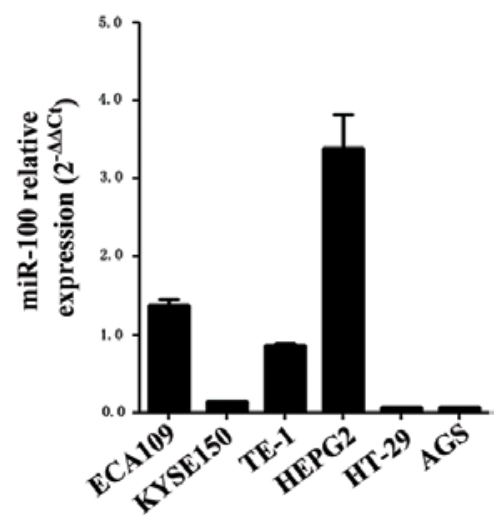

Figure 1. (A) Microarray analysis revealed that the expression of miR-100 in ESCC tissues was lower when compared with that in adjacent normal esophageal epithelial tissues. (B) qRT-PCR confirmed that the miR-100 relative expression level was decreased in 34 cases of ESCC tissues when compared with that in the adjacent normal esophageal epithelial tissues $(\mathrm{P}<0.01)$. (C) Expression of miR-100 in 3 ESCC cell lines and 3 gastrointestinal tract cancer cell lines. ESCC, esophageal squamous cell carcinoma.

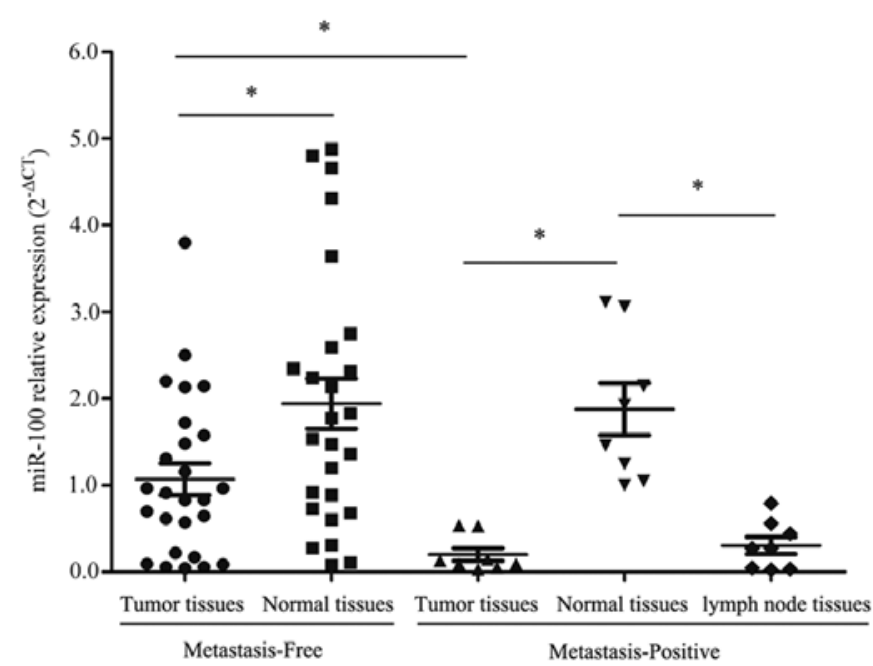

Figure 2. Relationship between miR-100 expression levels and tumor metastasis. Tumor tissues exhibited lower expression of miR-100 than adjacent normal tissues $(\mathrm{P}<0.05)$. The relative expression of miR-100 was significantly decreased in tumor tissues with lymph node metastasis compared with tissues from patients without lymph node metastasis $(\mathrm{P}<0.05)$. The tumor tissues and lymph node tissues had obviously decreased miR-100 expression compared with adjacent normal tissues in the metastasis-positive group $(\mathrm{P}<0.05)$.

sion of miR-100 in the ESCC and gastrointestinal tract cancer cell lines. The results showed that the relative expression of miR-100 was highest in the HEPG2 cell line, with the lowest levels of expression in the HT-27 and AGS cell lines and the relative expression of the three ESCC cell lines from highest to lowest is as follows: ECA109, TE-1, KYSE150 (Fig. 1C).

Association of miR-100 expression with metastasis of ESCC. The relationship between the relative expression of miR-100 and the clinicopathological features of the ESCC patients was analyzed. The tumor tissues showed a lower relative expression of miR-100 when compared with that in the adjacent normal tissues; the relative expression of miR-100 was significantly
Table I. Characteristics of the ESCC patients.

\begin{tabular}{lrc}
\hline $\begin{array}{l}\text { Clinicopathological } \\
\text { features }\end{array}$ & $\begin{array}{c}\text { Case } \\
\text { distribution }\end{array}$ & $\begin{array}{c}\text { miR-100 expression } \\
\text { (mean } \pm \text { SD) }\end{array}$ \\
\hline $\begin{array}{l}\text { Age (years) } \\
\leq 60\end{array}$ & 18 & $0.540 \pm 0.381$ \\
$>60$ & 16 & $0.446 \pm 0.319$ \\
Gender & & \\
Male & 27 & $0.493 \pm 0.359$ \\
Female & 7 & $0.509 \pm 0.348$ \\
Grade of differentiation & & \\
Well & 11 & $0.343 \pm 0.210$ \\
Moderate & 17 & $0.588 \pm 0.437$ \\
Poor & 5 & $0.455 \pm 0.261$ \\
Degree of tumor invasion & & \\
Submucosa & 3 & $0.501 \pm 0.361$ \\
Muscularis propria & 30 & $0.313 \pm 0.057$ \\
Adventitia & 1 & 0.576 \\
Lymph node metastasis & & $0.908 \pm 0.495$ \\
Negative & 26 & \\
Positive & 8 & \\
\hline
\end{tabular}

ESCC, esophageal squamous cell carcinoma.

decreased in the tumor tissues with lymph node metastasis compared to that in the tissues of the patients without lymph node metastasis. Meanwhile, the miR-100 expression in the normal tissues and the lymph node tissues was lower than that in the adjacent normal tissues in the metastasis-positive group, but no diffference was noted in the normal tissues and the lymph node tissues (Fig. 2). No significant association was found between miR-100 expression and the other clinical characteristics such as age, gender and grade of differentiation (Table I). 
A

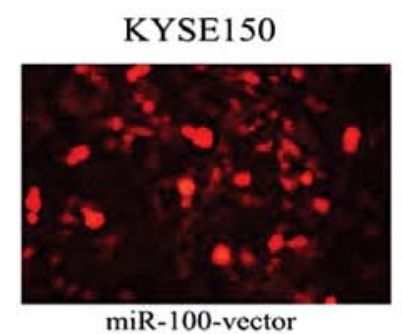

miR-100-vector

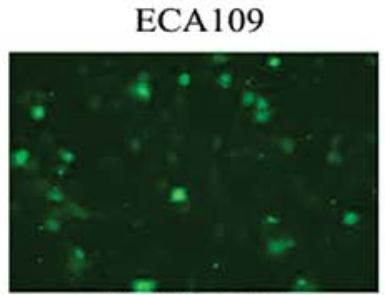

miR-100-inhibitor
$\mathrm{C}$

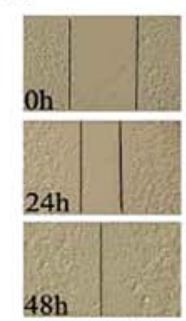

miR-100-inhibitor

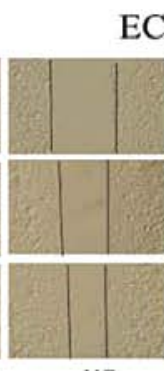

NC
ECA109

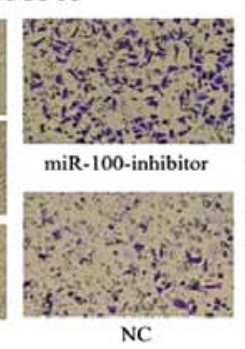

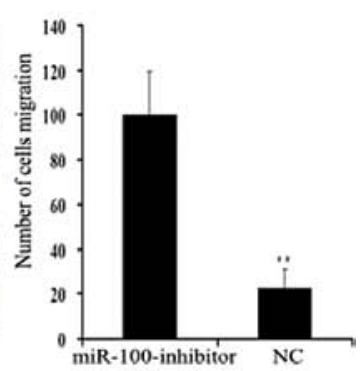

B

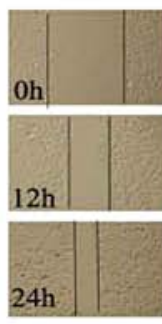

miR-100-vector

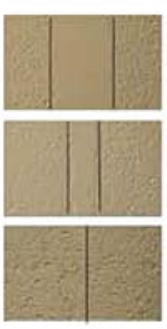

control
KYSE150

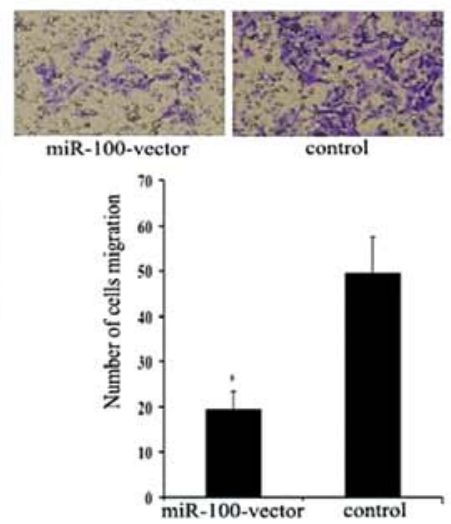

D
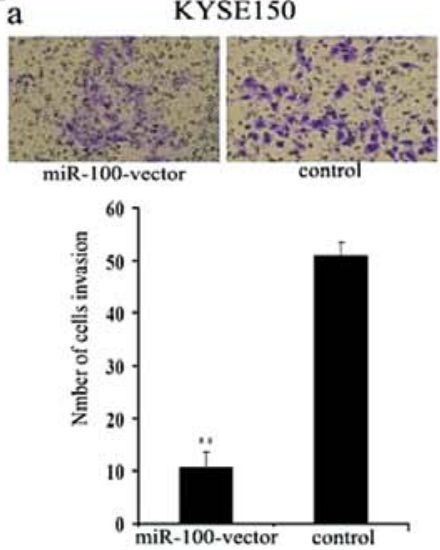

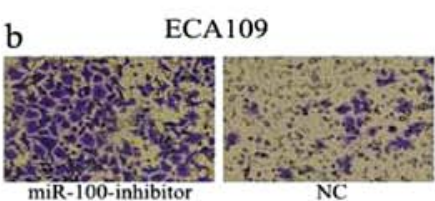

miR-100-inhibitor

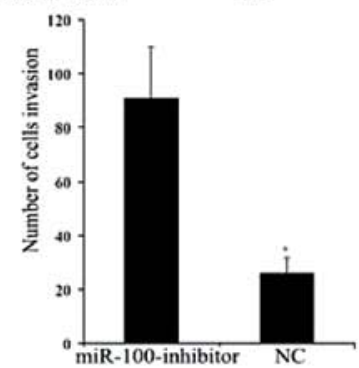

Figure 3. (A) Transfection efficiency of the miR-100-vector and miR-100-inhibitor in KYSE150 and ECA109 cell lines. (B) Wound healing assay showed that cell motility could be effectively suppressed by miR-100 overexpression in KYSE150 cells. Representative images were captured at time 0,24 and 48 h after scratching. Transwell assay showed that miR-100 could successfully impede KYSE150 cell metastasis (P<0.05). (C) Effect of miR-100 knockdown on ECA109 cell migration showed contrary results compared to the migration of overexpressed cells $(\mathrm{P}<0.01)$. (D) (a) Transwell assay revealed that cell invasion could be effectively suppressed by miR-100 overexpression in KYSE150 cells $(\mathrm{P}<0.01)$. (b) miRNA-100 knockdown significantly enhanced ECA109 cell invasion $(\mathrm{P}<0.05)$. The results are expressed as mean $\pm \mathrm{SD}$ of three independent experiments.

miR-100 modulates cellular migration and invasion of ESCC. To investigate the mechanism by which miR-100 inhibits lymph node metastasis, we examined the role of miR-100 in ESCC cell (KYSE150 and ECA109) migration and invasion. Since the relative expression of miR-100 was lower in the KYSE150 cell line compared with ECA109, we induced overexpression of miR-100 in the KYSE150 cells and knocked down miR-100 expression in the ECA109 cells (Fig. 3A). The expression of miR-100 was markedly increased by transfection of the miR-100-vector into KYSE150 cells and decreased by transfection of the miR100-inhibitor into ECA109 cells. Overexpression of miR-100 markedly decreased the migration of KYSE150 cells compared with the negative control $(\mathrm{P}<0.05)$ (Fig. 3B). Conversely, the inhibition of miR-100 expression led to considerable enhancement of the migration of ECA109 cells compared with the negative control $(\mathrm{P}<0.01)$ (Fig. 3C). Next, we examined the effect of miR-100 on cell invasion across an extracellular matrix and showed that in KYSE150 cells, overexpression of miR-100 markedly decreased the potential of invasion compared with the control $(\mathrm{P}<0.01)$ (Fig. 3D-a). However, knockdown of miR-100 in the ECA109 cells markedly enhanced the potential of invasion compared with the control $(\mathrm{P}<0.05)$ (Fig. 3D-b). These results demonstrate that miR-100 inhibits ESCC cell migration and invasion.

miRNA-100 is not involved in cell proliferation or apoptosis of ESCC. Next, the CCK-8 assay was used to analyze the prolif- eration of ESCC cells (KYSE150, ECA109) after transfection with the $\mathrm{miR}-100$-vector/miR-100-inhibitor and the negative controls at 12,24,48 and $72 \mathrm{~h}$ (Fig. 4A and B). The data indicated that the expression of miR-100 had no significant effect on the proliferation of ESCC cells ( $\mathrm{P}>0.05)$. In addition, analysis of apoptosis was carried out in the KYSE-150 and ECA109 cells by flow cytometry. In both ESCC cell lines, the data demonstrated that the overexpression and knockdown of miR-100 had no significant effect on the percentage of apoptotic cells, compared with the negative control ( $\mathrm{P}>0.05)$ (Fig. 4C and D).

miR-100 directly targets mTOR 3'UTR. The abiliity of miR-100 to promote cell migration and invasion may be due to its ability to regulate the expression of genes related to metastatic dissemination. Thus, we utilized bioinformatic analyses available on the Web, including miRBase, NCBI mapviewer and UCSC Genome Browser. The analysis results showed that miR-100 was highly conserved among species and hsa-miR100 is located on chromosome 11q24.1. Pictar, TargetScan, miRanda and miRBase were used to analyze putative miR-100 targets that may contribute to metastasis. Thousand of genes have been reported that are involved in tumor proliferation, migration and invasion in our predicted results, and we synthetically analyzed the intersection of respective predictive results. We found that predicted target genes of miR-100, included mTOR, FZD8, IGF1R and FGFR3 (Fig. 5A and B), which are relative to tumor metastasis, whereas mTOR and 
A

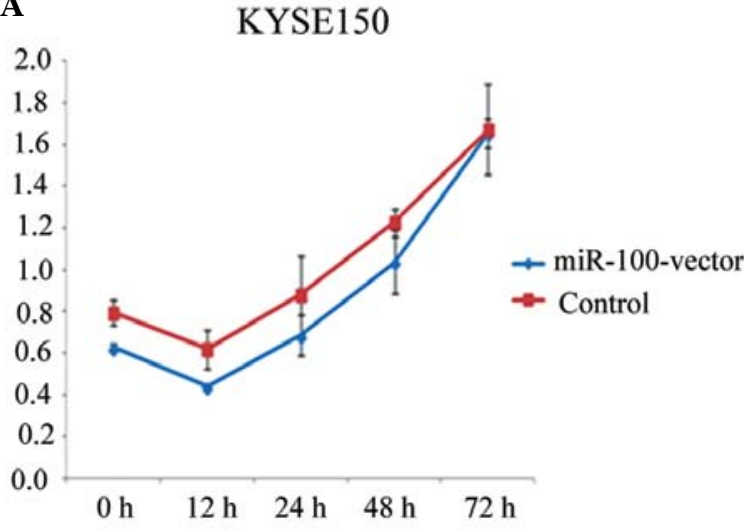

C

\section{KYSE150}

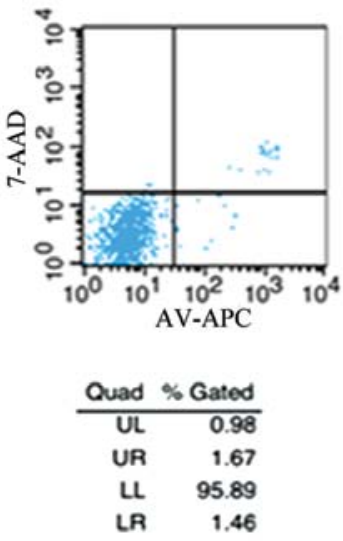

miR-100-vector

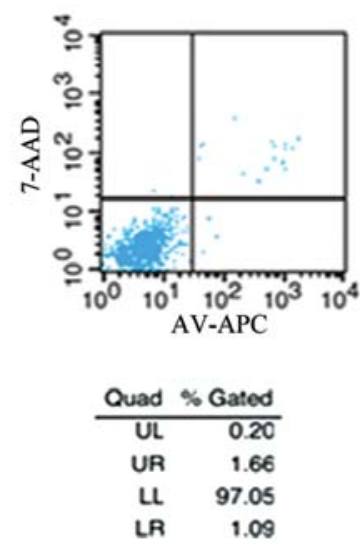

Control

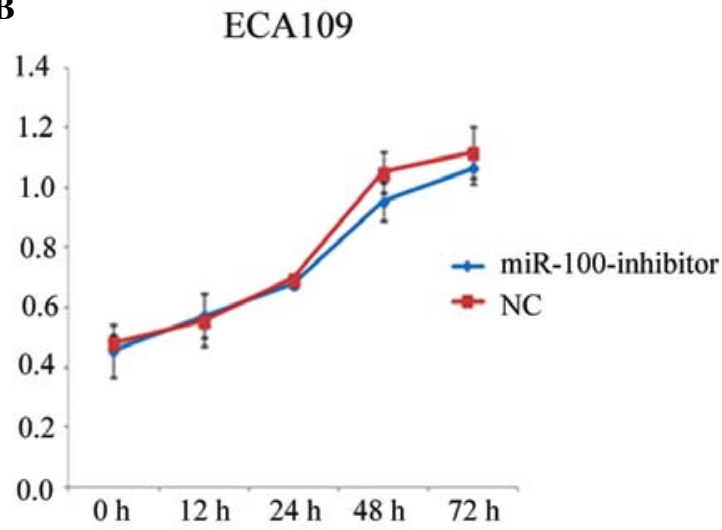

D

\section{ECA109}

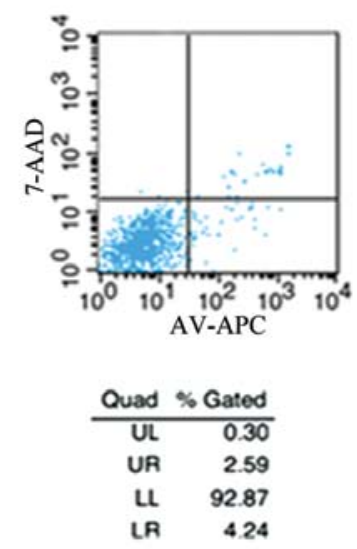

miR-100-inhibitor
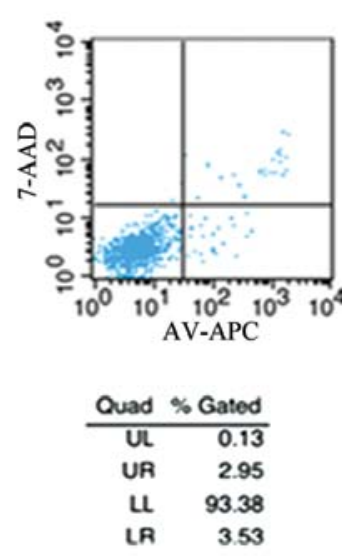

$\mathrm{NC}$

Figure 4. (A and B) The CCK-8 proliferation assay data indicated that miR-100 overexpression or knockdown had no significant effect on the proliferation of KYSE150 and ECA109 cells (P>0.05). (C and D) FACS analysis showed that miR-100 overexpression or knockdown had no significant effect on the apoptosis of KYSE150 and ECA109 cells (P>0.05). CCK-8, Cell Counting Kit-8.

IGF1R have been reported to be associated with ESCC. We then explored the expression levels of mTOR and IGF1R after transfection with the miR-100-vector or miR-100-inhibitor in ECA109 cells. Western blot assay showed that the overexpression of miR-100 significantly decreased the level of mTOR protein. Conversely, the level of mTOR protein was markedly upregulated by suppression of miR-100 expression (Fig. 5C). To further investigate whether miR-100 directly targets the 3'UTR of mTOR mRNA, we performed a dual-luciferase reporter assay. Cotransfection of the reporter plasmid along with the miR-100 precursor resulted in a significantly reduced mTOR-3'UTR-wt luciferase expression. In the reporter assay using the mTOR-3'UTR-mt, however, the miR-100 precursor was unable to reduce the luciferase reporter expression (Fig. 5D). This indicates that the 3'UTR of mTOR mRNA is a direct target of miR-100.

Immunohistochemistry (IHC). IHC was conducted to examine mTOR and IGF1R expression levels in human ESCC tissue specimens. We found elevated levels of mTOR in the tumor tissues compared to levels in the normal tissues. Moreover, we found that the IGF1R expression was also increased in the ESCC tissue specimens, but it was lower than that of mTOR (Fig. 6).

\section{Discussion}

Esophageal cancer (EC) is a common cause of cancer-related death worldwide. Esophageal squamous cell carcinoma (ESCC) is the most predominant type of EC, particularly in China. According to data from the Disease Control and Prevention Center of Jiangsu Province, cancer was the leading cause of mortality among Jiangsu inhabitants in 2007, and EC ranked first as the leading cause of cancer-related mortality (21). Huaian in Jiangsu Province is an area with high ESCC morbidity and mortality rates. Specific genes of the population in this area and the geographical environment may be related to various distinguishing features of ESCC. Although the local government has taken various measures to minimize ESCC risk factors, such as lifestyle changes, particularly the avoidance of tobacco and alcohol use and the elimination of high salt and moldy foods, the incidence and mortality have not significantly changed. The reason may be related to the uncertainty of the exact etiology and pathogenesis of ESCC as well as the lack of indicators for early diagnosis.

miRNAs are a class of small non-coding RNA molecules which can regulate gene expression at the post-transcriptional level by targeting mRNAs (22). Data analysis for bioinformatics 

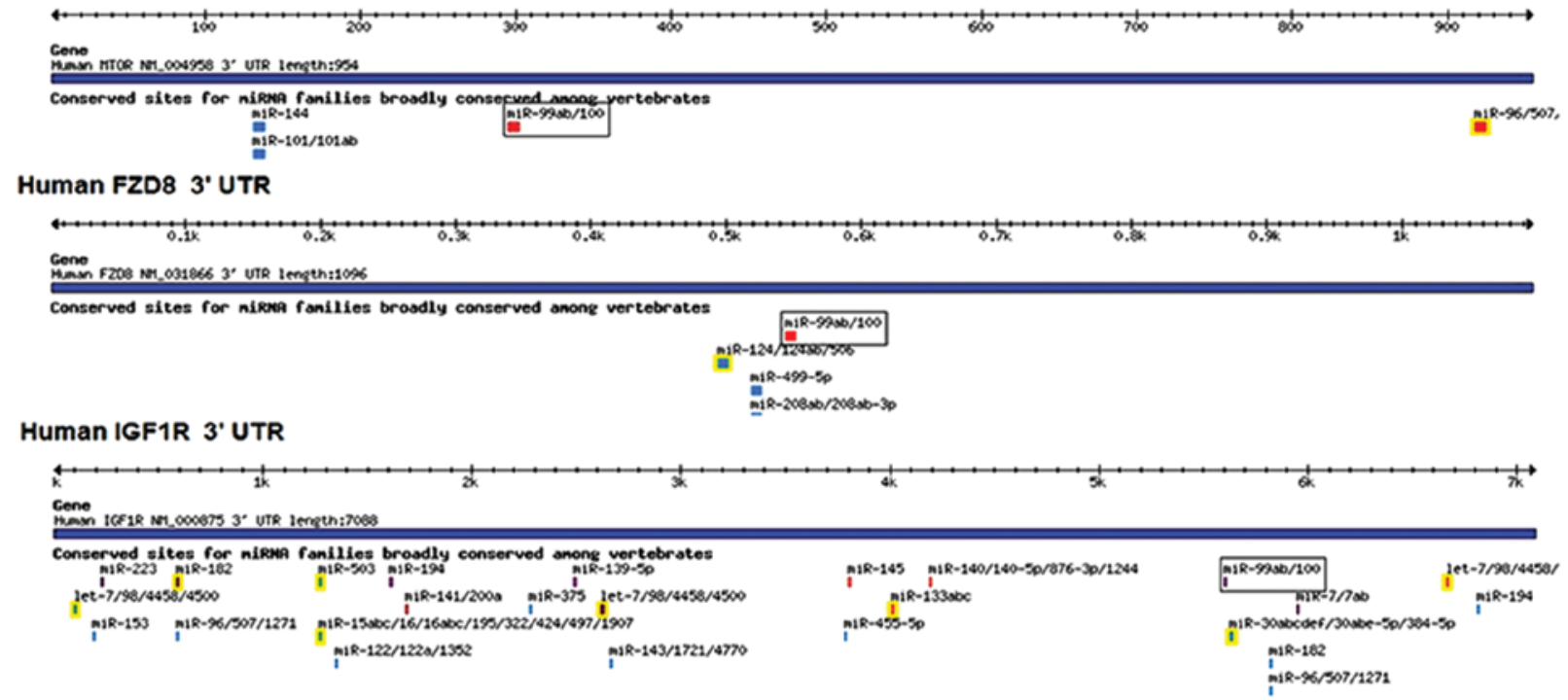

Human FGFR3 3. UTR

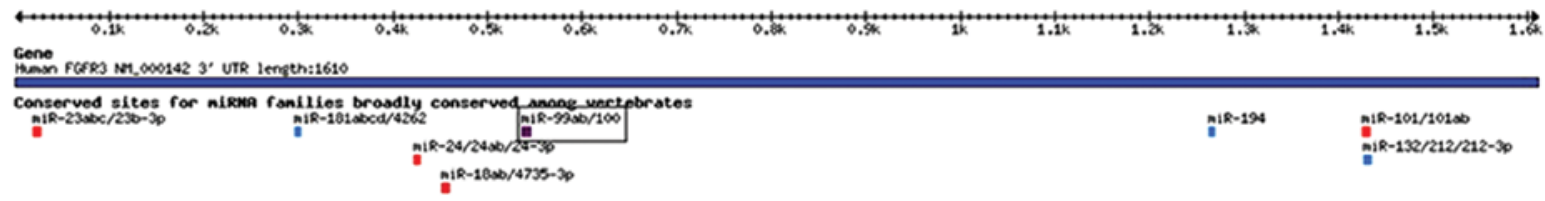

B

$\begin{aligned} \text { Position 295-301 of MTOR 3'UTR } & 5 \text { '...CCAUAACUUUAGAAAUACGGGUU... } \\ \text { hsa-miR-100 } & \text { 3' } \quad \text { GUGUUCAAGCCUAGAUGCCCAA } \\ \text { Position 544-550 of FZD8 3'UTR } & 5 \text { '...UACACCCCACGUAAAUACGGGUU... } \\ \text { hsa-miR-100 } & 3 \text { ' GUGUUCAAGCCUAGAUGCCCAA } \\ \text { Position 5602-5609 of IGFIR 3' UTR } & 5 \text { '...UUAAGUCCAGUAGAUUACGGGUA... } \\ \text { hsa-miR-100 } & \text { 3' GUGUUCAAGCCUAGAUGCCCAA } \\ \text { Position 537-544 of FGFR3 3'UTR } & 5 \text { '...CUCAGAGACUGAAAUUACGGGUA... } \\ \text { hsa-miR-100 } & \text { 3' GUGUUCAAGCCUACAUGCCCAA }\end{aligned}$

$\mathbf{C}$

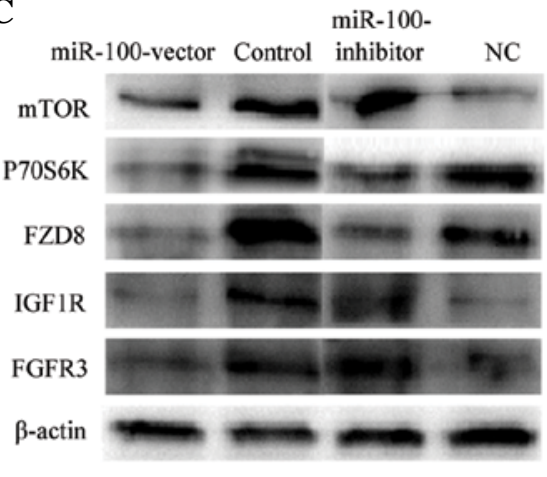

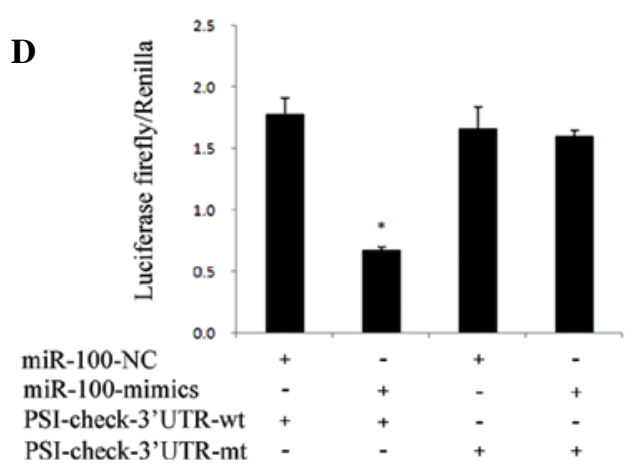

Figure 5. Human miR-100 targets and its binding sites predicted by the TargetScan program. (A) Human genes mTOR, FZD8, IGF1R, FGFR3 and their conserved binding sites for miR-100. (B) The specific sequences and positions of the binding sites pairing with miR-100 in 3'UTR of mTOR, FZD8, IGF1R, FGFR3. (C) Western blot assay showed that overexpression of miR-100 could significantly downregulate the level of mTOR protein and the level of mTOR protein was markedly upregulated by suppressing the miR-100 expression in ECA109 cells. However the expression of miR-100 did not obviously upregulate or downregulate FZD8, IGF1R and FGFR3 protein expression. (D) Luciferase assays showing decreased reporter activity after cotransfection of wild-type mTOR 3'UTR with miR-100 in HEK293 cells.

has demonstrated that the expression of approximately one-third of all human messenger RNAs is potentially regulated by miRNAs, including many important cancer-related genes (23). The role of miRNAs in the pathogenesis of ESCC is being taken into account. Hiyoshi et al showed that the expression levels of miR-21 were notable increased in ESCC tissues compared to levels in adjacent non-tumor tissues (24). In a Chinese population from Beijing, Guo et al identified seven miRNAs that 


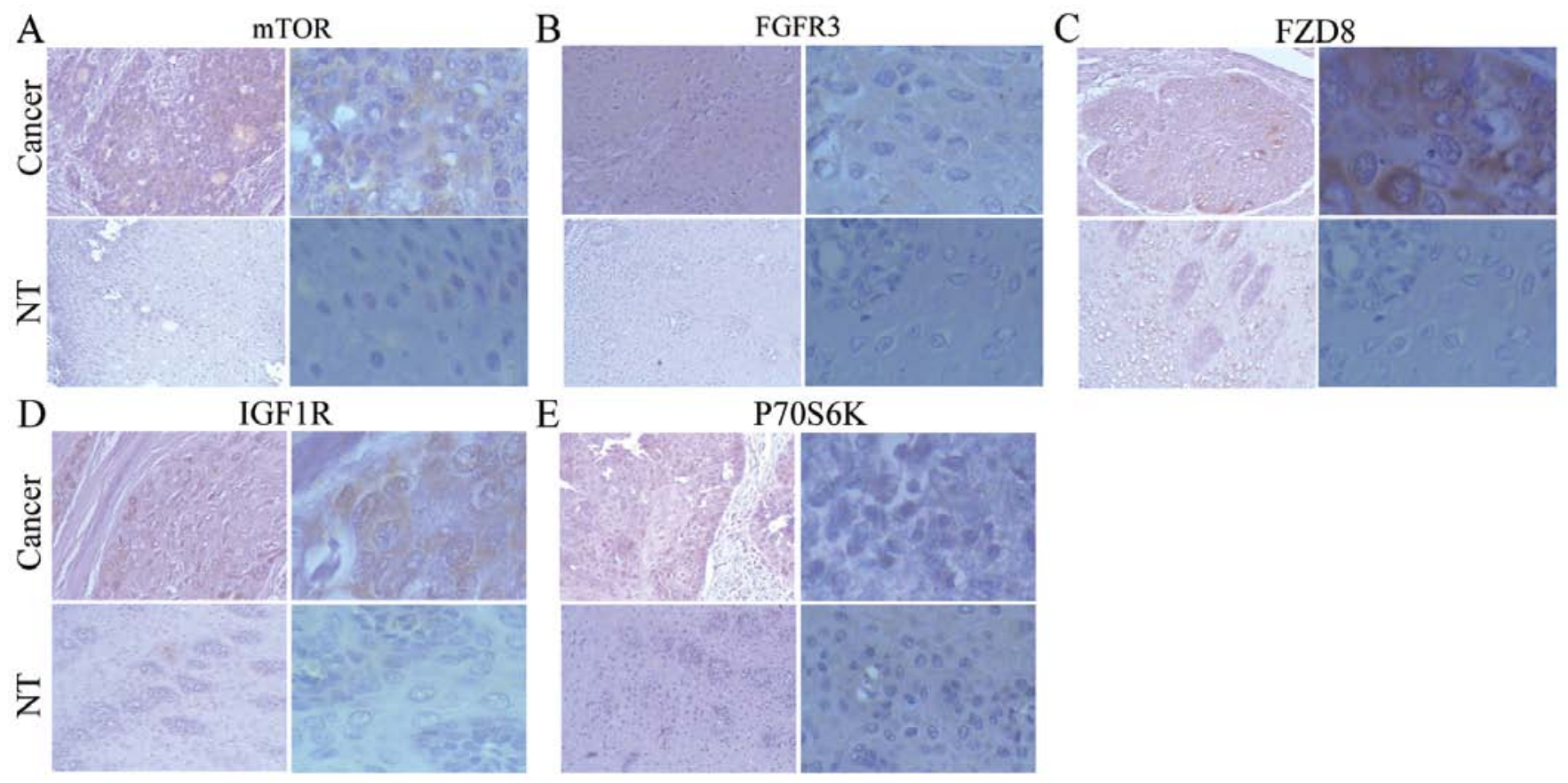

Figure 6. Representative cases of immunohistochemical assay of (A) mTOR, (B) FGFR3, (C) FZD8, (D) IGF1R and (E) p70 S6 kinase (P70S6K) in FFPE samples; the results showed that mTOR is positive in ESCC tissues. (Top) ESCC tissues (left panel x10, right panel x40). (Bottom) Adjacent normal esophageal epithelial tissues (NT), (left panel x10, right panel x40). ESCC, esophageal squamous cell carcinoma.

could distinguish ESCC lesions from adjacent normal tissues. Among the seven miRNAs, four miRNAs (miR-100, miR-99a, miR-29c and miR-140*) were downregulated and three miRNAs (miR-25, miR-424 and miR-151) were upregulated in cancer tissues when compared with normal tissues (25). Smith et al found reduced miR-375 expression levels and elevated miR-21 levels in patients with ESCC from the US (26). Our previous study found that the expression levels of miRNA-1, miRNA-29c, miR-100, miRNA-133a, miRNA-133b, miRNA143, miRNA-145 and miRNA-195 were significantly decreased in ESCC, while miRNA-7, miRNA-21, miRNA-223 and miRNA-1246 were significantly increased (13). Thus, miRNA expression profiling of ESCC displays obvious differences between different geographical regions.

Invasion and metastasis are important factors in the survival of ESCC, yet the molecular mechanisms are unclear. It has been reported that miRNAs are involved in tumor invasion and metastasis and miR-100 has been reported to be involved in tumorigenesis and tumor progression of several cancer types, including prostate and lung cancer, acute myeloid leukemia, epithelial ovarian, bladder, oral and breast cancer. It has been reported that miRNA levels are significantly correlated with the migration and invasion potential of cancer. Kinoshita et al showed that tumor suppressive miR-218 contributes to head and neck squamous cell carcinoma cell migration and invasion through regulation of laminin-332 (27). Majid et al found that miR-1280 functions as a tumor suppressor by targeting oncogene ROCK1 promoting invasion/migration and metastasis in bladder cancer (28).

To determine whether miR-100 is involved in the invasion and metastasis of ESCC, two ESCC cell lines ECA109 (well differentiated) and KYSE150 (poorly differentiated) were selected as test material in this study. The results revealed that the expression of miR-100 had a regulatory function in the various differentiated ESCC cell lines. Overexpression of miR-100 significantly inhibited the migration and invasion of ESCC cells, while knockdown promoted the migration and invasion of ESCC cells. The expression of miR-100 was significantly lower in positive lymph node metastatic ESCC tissues than that in the negative group. Thus, we speculated that miR-100 may play an important role in ESCC progression, in the Huaian region.

Abnormal regulation of cell proliferation and apoptosis is thought to be involved in tumor formation. It has been reported that miR-100 regulates G1/S transition and S-phase entry and blocks terminal differentiation by targeting RBSP3 in acute myelocytic leukemia (AML) (16). Liu et al found that miR-100 was upregulation in medulloblastoma as determined by high throughput microRNA microarray (29) and acts on its target genes ATAD2, BTG2, CASP8, CC2D1A, HOXA1, INSM1, MTMR3, NDRG1, SUFU and PTCH1 to regulate the cell cycle, cell proliferation and apoptosis. Li et al demonstrated that the expression level of miR-100 was closely related to cell proliferation (30), apoptosis and the cell cycle in cervical cancer. Our studies showed that overexpression or downregulation of the expression of miR-100 have no regulatory function on proliferation and apoptosis in well or poorly differentiated ESCC cell lines. miRNAs are important factors for regulating gene expression and this regulation is reflected in many aspects. One miRNA may regulate multiple target genes and one target gene can be regulated by a number of miRNAs, thus it may produce a complex and large regulatory network. One miRNA can regulated different target genes that play a different role in various disease, and our results indicate that the proliferation and apoptosis may not be the primary mechanisms for ESCC occurrence where miR-100 is involved.

Collectively, these results and the related literature suggest that the mTOR gene in the PI3K/AKT pathway may be the 
target gene of miR-100. The PI3K/AKT pathway is believed to be closely related to the tumorigenesis and development of cancer (31-33). Research shows that there are many genes involved in the PI3K/AKT pathway with abnormal expression in ESCC tissue and cell lines $(34,35)$. Thus, we believe that miR-100 could regulate the key protein mTOR in the PI3K/AKT signaling pathway involved in the mechanism of ESCC. mTOR is a central signal integrator that receives signals arising from growth factors, nutrients and a central controller of eukaryotic cell growth and proliferation (36-38). It has been demonstrated that activated mTOR has many functions not only for growth but also for invasion and metastasis of tumors (39-41).

In the present study, western blot assay demonstrated that overexpression of miR-100 decreased the expression of mTOR at the protein level. In addition, inhibiting miR-100 expression increased mTOR expression. Luciferase assay showed that miR-100 could interact with the 3'UTR of mTOR. Moreover, immunohistochemistry and qRT-PCR analysis indicated that the expression of mTOR was obviously increased and miR-100 expression was decreased in clinical ESCC specimens. These data strongly suggest that the tumor suppressive function of miR-100 is through the downregulation of mTOR, which may have a strong implication for the PI3K/AKT pathway in ESCC.

In conclusion, we identified that miR-100 expression is obviously downregulated in patients with ESCC from Huaian, China. miR-100 was found to be involved in invasion and metastasis of ESCC and plays a suppressive role through regulation of mTOR which is involved in the PI3K/AKT pathway and is obviously upregulated in ESCC. Our data present new evidence for the important roles of miR-100 in ESCC, and lay the foundation for further study in human ESCC.

\section{Acknowledgements}

The present study was supported by the Natural Science Foundation of Jiangsu Province, China (BK2012666).

\section{References}

1. Enzinger PC and Mayer RJ: Esophageal cancer. N Engl J Med 349: 2241-2252, 2003.

2. Orringer MB: Multimodality therapy for esophageal carcinomaupdate. Chest 103: 406S-409S, 1993.

3. Rice TW, Rusch VW, Apperson-Hansen C, et al: Worldwide esophageal cancer collaboration. Dis Esophagus 22: 1-8, 2009.

4. Bartel DP: MicroRNAs: genomics, biogenesis, mechanism, and function. Cell 116: 281-297, 2004.

5. Lewis BP, Burge CB and Bartel DP: Conserved seed pairing, often flanked by adenosines, indicates that thousands of human genes are microRNA targets. Cell 120: 15-20, 2005.

6. Lu J, Getz G, Miska EA, et al: MicroRNA expression profiles classify human cancers. Nature 435: 834-838, 2005.

7. Calin GA and Croce CM: MicroRNA signatures in human cancers. Nat Rev Cancer 6: 857-866, 2006.

8. Kong YW, Ferland-McCollough D, Jackson TJ and Bushell M: microRNAs in cancer management. Lancet Oncol 13: e249-e258, 2012.

9. Jia XQ, Cheng HQ, Qian X, et al: Lentivirus-mediated overexpression of microRNA-199a inhibits cell proliferation of human hepatocellular carcinoma. Cell Biochem Biophys 62: 237-244, 2012.

10. Calin GA, Sevignani C, Dumitru CD, et al: Human microRNA genes are frequently located at fragile sites and genomic regions involved in cancers. Proc Natl Acad Sci USA 101: 2999-3004, 2004

11. Kong KL, Kwong DL, Chan TH, et al: MicroRNA-375 inhibits tumour growth and metastasis in oesophageal squamous cell carcinoma through repressing insulin-like growth factor 1 receptor. Gut 61: 33-42, 2012.
12. Zhang HF, Zhang $\mathrm{K}$, Liao LD, et al: miR-200b suppresses invasiveness and modulates the cytoskeletal and adhesive machinery in esophageal squamous cell carcinoma cells via targeting Kindlin-2. Carcinogenesis 35: 292-301, 2014.

13. Fu HL, Wu de P, Wang XF, et al: Altered miRNA expression is associated with differentiation, invasion, and metastasis of esophageal squamous cell carcinoma (ESCC) in patients from Huaian, China. Cell Biochem Biophys 67: 657-668, 2013.

14. Leite KR, Tomiyama A, Reis ST, Sousa-Canavez JM, Sanudo A, Camara-Lopes LH and Srougi M: MicroRNA expression profiles in the progression of prostate cancer - from high-grade prostate intraepithelial neoplasia to metastasis. Urol Oncol 31: 796-801, 2013.

15. Feng B, Wang $\mathrm{R}$ and Chen LB: MiR-100 resensitizes docetaxel-resistant human lung adenocarcinoma cells (SPC-A1) to docetaxel by targeting Plk1. Cancer Lett 317: 184-191, 2012

16. Zheng YS, Zhang $\mathrm{H}$, Zhang XJ, et al: MiR-100 regulates cell differentiation and survival by targeting RBSP3, a phosphatase-like tumor suppressor in acute myeloid leukemia. Oncogene 31: 80-92, 2012.

17. Peng DX, Luo M, Qiu LW, He YL and Wang XF: Prognostic implications of microRNA-100 and its functional roles in human epithelial ovarian cancer. Oncol Rep 27: 1238-1244, 2012.

18. Oliveira JC, Brassesco MS, Morales AG, et al: MicroRNA-100 acts as a tumor suppressor in human bladder carcinoma 5637 cells. Asian Pac J Cancer Prev 12: 3001-3004, 2011.

19. Henson BJ, Bhattacharjee S, O'Dee DM, Feingold E and Gollin SM: Decreased expression of miR-125b and miR-100 in oral cancer cells contributes to malignancy. Genes Chromosomes Cancer 48: 569-582, 2009.

20. Gebeshuber CA and Martinez J: miR-100 suppresses IGF2 and inhibits breast tumorigenesis by interfering with proliferation and survival signaling. Oncogene 32: 3306-3310, 2013.

21. Zhu Q, Hong A, Sheng N, et al: microParaflo biochip for nucleic acid and protein analysis. Methods Mol Biol 382: 287-312, 2007.

22. Hutvagner G, McLachlan J, Pasquinelli AE, Balint E, Tuschl T and Zamore PD: A cellular function for the RNA-interference enzyme Dicer in the maturation of the let-7 small temporal RNA. Science 293: 834-838, 2001.

23. Alvarez-Garcia I and Miska EA: MicroRNA functions in animal development and human disease. Development 132: 4653-4662, 2005.

24. Hiyoshi Y, Kamohara H, Karashima R, et al: MicroRNA-21 regulates the proliferation and invasion in esophageal squamous cell carcinoma. Clin Cancer Res 15: 1915-1922, 2009.

25. Guo Y, Chen Z, Zhang L, et al: Distinctive microRNA profiles relating to patient survival in esophageal squamous cell carcinoma. Cancer Res 68: 26-33, 2008.

26. Smith CM, Watson DI, Michael MZ and Hussey DJ: MicroRNAs, development of Barrett's esophagus, and progression to esophageal adenocarcinoma. World J Gastroenterol 16: 531-537, 2010.

27. Kinoshita T, Hanazawa T, Nohata N, et al: Tumor suppressive microRNA-218 inhibits cancer cell migration and invasion through targeting laminin-332 in head and neck squamous cell carcinoma. Oncotarget 3: 1386-1400, 2012.

28. Majid S, Dar AA, Saini S, et al: MicroRNA-1280 inhibits invasion and metastasis by targeting ROCK1 in bladder cancer. PLoS One 7: e46743, 2012.

29. Liu W, Gong YH, Chao TF, et al: Identification of differentially expressed microRNAs by microarray: a possible role for microRNAs gene in medulloblastomas. Chin Med J 122: 2405-2411, 2009

30. Li BH, Zhou JS, Ye F, Cheng XD, Zhou CY, Lu WG and Xie X: Reduced miR-100 expression in cervical cancer and precursors and its carcinogenic effect through targeting PLK1 protein. Eur J Cancer 47: 2166-2174, 2011.

31. Hildebrandt MA, Yang H, Hung MC, et al: Genetic variations in the PI3K/PTEN/AKT/mTOR pathway are associated with clinical outcomes in esophageal cancer patients treated with chemoradiotherapy. J Clin Oncol 27: 857-871, 2009.

32. Oyama K, Okawa T, Nakagawa H, et al: AKT induces senescence in primary esophageal epithelial cells but is permissive for differentiation as revealed in organotypic culture. Oncogene 26: 2353-2364, 2007.

33. Okano J, Gaslightwala I, Birnbaum MJ, Rustgi AK and Nakagawa H: Akt/protein kinase B isoforms are differentially regulated by epidermal growth factor stimulation. J Biol Chem 275: 30934-30942, 2000. 
34. Baba Y, Iyama KI, Hirashima K, et al: Laminin-332 promotes the invasion of oesophageal squamous cell carcinoma via PI3K activation. Br J Cancer 98: 974-980, 2008.

35. Li B, Tsao SW, Li YY, et al: Id-1 promotes tumorigenicity and metastasis of human esophageal cancer cells through activation of PI3K/AKT signaling pathway. Int J Cancer 125: 2576-2585, 2009.

36. Hay N and Sonenberg N: Upstream and downstream of mTOR Genes Dev 18: 1926-1945, 2004

37. Kim JE and Chen J: Cytoplasmic-nuclear shuttling of FKBP12rapamycin-associated protein is involved in rapamycin-sensitive signaling and translation initiation. Proc Natl Acad Sci USA 97: 14340-14345, 2000.

38. Martin DE and Hall MN: The expanding TOR signaling network. Curr Opin Cell Biol 17: 158-166, 2005.
39. Sun SY, Rosenberg LM, Wang X, Zhou Z, Yue P, Fu H and Khuri FR: Activation of Akt and eIF4E survival pathways by rapamycin-mediated mammalian target of rapamycin inhibition. Cancer Res 65: 7052-7058, 2005.

40. Treeck O, Wackwitz B, Haus U and Ortmann O: Effects of a combined treatment with mTOR inhibitor RAD001 and tamoxifen in vitro on growth and apoptosis of human cancer cells. Gynecol Oncol 102: 292-299, 2006.

41. DeGraffenried LA, Friedrichs WE, Russell DH, et al: Inhibition of mTOR activity restores tamoxifen response in breast cancer cells with aberrant Akt activity. Clin Cancer Res 10: 8059-8067, 2004. 